“ (C) 2017 IEEE. Personal use of this material is permitted. Permission from IEEE must be obtained for all other uses, in any current or future media, including

reprinting/republishing this material for advertising or promotional purposes, creating new collective works, for resale or redistribution to servers or lists, or reuse of any copyrighted component of this work in other works." 


\title{
Cancer Detection in Highly Dense Breasts using Coherently Focused Time Reversal Microwave Imaging
}

\author{
Md. Delwar Hossain, Member, IEEE and Ananda Sanagavarapu Mohan, Senior Member, IEEE
}

\begin{abstract}
Detection of tumors in highly dense breasts is a critical but challenging issue for early stage breast cancer detection. We present the application of coherent focusing for time reversal (TR) microwave imaging in beamspace for the detection and localization of multiple tumors in highly dense, 3-D breast phantoms. We propose a novel Coherent-Beamspace-Time Reversal-Maximum Likelihood (C-B-TR-ML) technique to obtain accurate tumor locations with reduced computational burden. To compare the performance, the coherent beamspace processing is also extended for conventional Decomposition of the Time Reversal Operator (DORT) and Time Reversal MUSIC (TR-MUSIC) algorithms. A novel hybrid technique involving time of arrival (TOA) and entropy is also proposed for early-time artifact removal as well as for estimating the Green's function of an equivalent virtual medium required for the time reversal operation. Finite difference time domain (FDTD) computations on anatomically realistic, 3-D numerical breast phantoms are used to obtain the backscattered data. The results demonstrate the superior capabilities of the proposed C-B-TR-ML microwave imaging technique in detecting and localizing multiple tumors embedded inside highly dense breast phantoms.
\end{abstract}

Index Terms - Beamspace, breast cancer, coherent focusing, DORT, entropy, Green's function, highly dense breasts, maximum likelihood, microwave imaging, multiple tumors, time of arrival, time reversal, TR-MUSIC.

\section{INTRODUCTION}

$\mathrm{B}$ REAST cancer is one of the most common life threatening diseases affecting women around the globe. Early detection of cancer can improve the chances of survival. The density of breast tissue in younger women often makes it difficult to detect cancers on mammograms [1]. The increased breast tissue density has been linked to an increased risk of cancer occurrence [2]. Other existing clinical breast screening modalities also have limitations for detecting malignancies in dense breasts [3]. Microwave imaging has demonstrated its potential to develop into a complementary modality for early stage breast cancer detection [4]. In this paper, we present

Manuscript submitted 13 March, 2017; revised 3 July, 2017; accepted 25 July, 2017. The work reported in this paper was originally initiated with the support from the Australian Research Council through a Discovery Project Grant DP 0773234 and later supported by Faculty of Engineering and IT, University of Technology Sydney (UTS), Bluesky Research Grant.

The authors are with the Centre for Health Technologies, Faculty of Engineering and IT, UTS, Australia (e-mail: Md.Hossain@uts.edu.au, Ananda.Sanagavarapu@uts.edu.au). novel computational time reversal (TR) techniques to further enhance the capabilities of microwave imaging for the detection of multiple, small sized malignancies in highly dense (class 4 ) and heterogeneously dense (class 3 ) breast phantoms.

The breasts consist of fatty adipose and dense fibroglandular and connective tissues. The Breast ImagingReporting and Data System (BIRADS) classifies breasts into four categories based on the amount of glandular tissue content present in the breast parenchyma. Microwave imaging makes use of the backscattering responses from the breast tissues which in turn depend on their dielectric properties. It has been well established that the dielectric property contrast between malignant and healthy fibroglandular breast tissues is quite modest, i.e., only around $10 \%$ [4]. This poses additional detection challenges in terms of discriminating weak tumor signature from the strong backscatter from healthy glandular tissues. This will be further accentuated by the path attenuation of the microwave signal as it propagates through the heterogeneous breast tissue medium [4-6]. This uncertainty can lead to detection ambiguities for any existing imaging modality, causing false positives or false negatives [7-9] and even more so, when multiple small tumors are present in the breast.

Different variations of microwave imaging techniques, viz., tomography [2, 10-14] and radar-based imaging [15-18] have been extensively investigated for breast cancer detection and some of which also underwent successful clinical trials $[12$, 17, 19, 20]. The low dielectric contrast can reduce the ability of radar based imaging to differentiate between tissues while the low resolution of microwave tomography can result in underestimation of the electrically small features of the tumors' dielectric profile $[2,12]$. These can potentially miss some of the small sized malignancies that are associated with early stage cancer [21]. Hybrid imaging modalities have been proposed $[8,22,23]$ to overcome these challenges. The use of contrast enhancing agents to raise the dielectric contrast between malignant lesion and healthy breast tissues has also been suggested [9, 21, 24-28]. However, to discriminate weak signals due to the tumor from clutter in such scenarios, require differential imaging technique whose sensitivity can be severely affected by the random alterations of dielectric tissue properties due to contrast agents as well as by the physiological movements of the patients $[9,26]$. To minimize this problem, the magnetic contrast modulation by a low 
frequency magnetic field has been suggested but the choice of the modulation frequency for filtering out physiological movements requires careful selection [24].

The TR imaging technique does not involve any of the above and is a likely contender for breast cancer detection [3035 ] as it is greatly suitable for target localization in heterogeneous media [29]. The localization performance of existing TR imaging techniques viz., DORT and TR-MUSIC deteriorate with an increase in the dense tissue content in the breast medium [32-34, 36], as they require the orthogonal decomposition of the time reversal operator (TRO). On the other hand, non-subspace based imaging techniques such as time reversal Robust Capon Beamformer (TR-RCB) and time reversal Maximum Likelihood (TR-ML) methods do not involve any decomposition of the TRO. The TR-RCB has successfully been employed for breast cancer detection [37], but its accuracy tends to suffer from the increase in the breast tissue density. Shi and Nehorai [38] proposed a time reversal maximum likelihood (TR-ML) imaging method for the detection of point targets embedded in an infinite twodimensional lossless dielectric medium. The TR-ML method can offer better performance as it obtains the signal subspace from the measurement data, unlike DORT and TR-MUSIC which mainly employ a hypothetical physical model. In spite of its inherent capabilities, the TR-ML proposed in [38] requires prohibitive computational load when directly extended for breast cancer detection in three-dimensional, heterogeneous, volumetric breast phantoms involving scattering from lossy, finite-sized, dielectric breast tissues under ultrawideband (UWB) microwave excitation. It would, therefore, be of interest to investigate techniques that can detect malignancies in highly dense breasts without the above mentioned limitations.

In this paper, we propose a novel Coherent-BeamspaceTime Reversal-Maximum Likelihood (C-B-TR-ML) imaging technique in anatomically realistic, 3-D breast phantoms for the detection of multiple tissue malignancies. Our proposed method combines the novel coherent focusing and beamspace processing with TR-ML for the image reconstruction. The coherent focusing process helps to overcome the challenges arising due to signal propagation in a highly cluttered heterogeneous breast medium. On the other hand, beamspace processing significantly reduces the computational burden of TR-ML imaging and in addition offers additional beamforming gain. Using numerical experiments on highly dense breast phantoms, we demonstrate the superior performance of the proposed technique for the detection and localization of multiple, small sized tumors. We also extend the coherent beamspace approach to the existing subspace based TR techniques to obtain C-B-DORT and C-BTRMUSIC whose imaging performances will be compared with that of C-B-TR-ML.

The paper is organized as follows. In section II, we describe the computational aspects of TR imaging in highly dense breasts to identify the challenges involved. In section III, we present the setup for numerical experiments for the forward problem along with the pre-processing methods employed.
The proposed coherent beamspace processing for TRO is introduced in section IV whereas the image reconstruction techniques are elaborated in section V. The efficacy of the proposed techniques is demonstrated through the computational results in section VI followed by conclusions in section VII. We adapt the following matrix/vector notation throughout this paper.
$(.)^{T}$ : Transpose of a vector or matrix
$(.)^{H}$ : Conjugate transpose of a vector or matrix
$(.)^{*}$ : Conjugate of a vector or matrix
$\operatorname{tr}($.$) : Trace of a matrix$
$\otimes \quad$ : Kronecker product

\section{Time ReVersal Imaging IN Highly Dense BREasts}

Computational TR operation is carried out in three basic steps- forward medium probing, time reversal of received response and then backpropagation of the time reversed signals into a computational (virtual) medium that closely resembles the original breast medium as illustrated in the Fig. 1. Conventional TR approaches operate in elementspace in which the received raw, output data from each element of an array of receivers (also known as the time reversal mirror), are processed. These received element by element responses, when time reversed and propagated back into the original medium, it is expected to be refocused in space and time at the actual location of the original target, assuming reciprocal, linear, and lossless medium. In the computational time reversal, an equivalent computational medium that closely resembles the original medium is employed for back propagation.

\begin{tabular}{|l|l|l|l|}
\hline $\begin{array}{l}\text { Forward } \\
\text { medium } \\
\text { probing }\end{array}$ & $\longrightarrow \begin{array}{l}\text { Time } \\
\text { reversal of } \\
\text { received } \\
\text { response }\end{array}$ & $\longrightarrow \begin{array}{l}\text { Backpropagation } \\
\text { of the time } \\
\text { reversed signals } \\
\text { into a } \\
\text { computational } \\
\text { medium }\end{array}$ \\
\hline
\end{tabular}

Fig. 1. Time reversal imaging process.

\section{A. Forward Problem}

A low power, radiated, UWB microwave probing pulse illuminates the breast and the resulting backscattered responses received at each element of the array are recorded in a multistatic data matrix. These raw, received responses include early time artifacts as well as the potential target signatures. Before proceeding further, it is critical to remove the early time artifacts from the recorded responses completely.

\section{1) Early time skin artifact}

The early time artifact is predominantly due to the reflections of the incident microwave pulse from the skin layer of the breast, as it is located physically close to the array. Thus, the magnitudes of skin reflections can be several orders of magnitude higher than the late-time backscattered signals from other internal breast tissues (parenchyma) which include any existing tissue malignancies. These late-time signals usually have weaker amplitudes as they undergo higher path 
attenuation due to traversing longer paths inside the lossy breast tissue medium. Thus, the early time artifacts must be removed entirely without distorting the late time responses for the successful detection of a tumor. To minimize the skin reflections, it is customary to immerse the breast into a matching liquid. However, this cannot ensure complete elimination of the skin reflections and separate early time artifact removal is mandated prior to the image reconstruction. In this paper, we propose a novel hybrid time of arrival (TOA) and entropy based signal processing technique for skin artifact removal which will be presented in section III.

\section{B. Time Reversal}

TR operation (last in first out) in the time domain is equivalent to phase conjugation in the frequency domain. The backscattering responses from targets embedded in a highly inhomogeneous breast medium are often frequency-selective. Hence, TR processing in the frequency domain can be prudent as it allows frequency binning [37, 39]. Also, use of UWB excitation helps to increase the stability of the TR process [40].

\section{1) Effects of Clutter}

The microwave backscattering response of a dielectric target embedded inside a background medium is a function of the contrast between their dielectric properties. In a highly heterogeneous dense breast medium, the responses from healthy, dense tissues act as clutter and it becomes extremely challenging to isolate target responses from the clutter.

Consider an $\mathrm{N}$-element transceiver antenna array to collect the multistatic responses from a bounded region containing $P$ targets $(P<N)$, the resulting multistatic matrix $\overline{\mathbf{K}}$ using Born approximation can be expressed as [41]

$$
\overline{\mathbf{K}}=\overline{\mathcal{G}} \chi \overline{\mathcal{G}} \overline{\mathcal{G}}^{T}
$$

where, $\overline{\mathcal{G}}$ is a $3 N \times 3 P$ matrix representing the Green's function vector and $\bar{\chi}$ is a $3 P \times 3 P$ matrix representing the scattering responses from the $P$ embedded targets. Here, we seek to perform the TR operation in the frequency domain.

The microwave scattering inside the breast is analogous to random multipath in a rich scattering medium where the target and clutter responses can be considered to be additive [42]. As a result, the responses received in individual frequency bins tend to be highly correlated. Hence, the signal subspace will no longer be uniform over the ultrawide bandwidth. For such a scenario, the received multistatic matrix in the frequency domain can be expressed as

$$
\overline{\mathbf{K}}_{b r}=\overline{\mathbf{K}}+\overline{\Delta \mathbf{K}}
$$

where, $\overline{\mathbf{K}}_{b r}$ is the recorded multistatic response matrix for a dense breast, $\overline{\mathbf{K}}$ is the ideal target (tumor) response in the absence of any clutter and $\overline{\Delta \mathbf{K}}$ represents combined effects due to clutter which tend to perturb the desired target response. Usually, the scattered field responses are independently recorded by individual array elements which are then combined incoherently in each bin before carrying out the time reversal operation. The expected image after the time reversal and back propagation is a bright focus at the actual location of the target. However, under high tissue clutter and low signal to noise ratio scenarios, such an incoherent imaging approach fails to localize the target accurately. Even when the signal to noise ratio is high, the correlation arising from clutter multipath among frequency bins can cause problems for focusing using incoherent imaging approach [43]. Thus, the incoherent time reversal imaging approach is inadequate for unambiguously localizing targets in a medium dominated by strong clutter. To further clarify, first consider the TRO that is formed using the multistatic matrix given by (2) as

$$
\begin{aligned}
\overline{\mathbf{T}}_{b r} & =\overline{\mathbf{K}}_{b r}^{H} \overline{\mathbf{K}}_{b r} \\
& =\overline{\mathbf{K}}^{H} \overline{\mathbf{K}}+\overline{\mathbf{K}}^{H} \overline{\Delta \mathbf{K}}+\overline{\Delta \mathbf{K}}^{H} \overline{\mathbf{K}}+\overline{\Delta \mathbf{K}}^{H} \overline{\Delta \mathbf{K}}^{H}
\end{aligned}
$$

The first term $\overline{\mathbf{K}}^{H} \overline{\mathbf{K}}$ on the right hand side of (3) is the perturbation-free TRO which is desirable, and the second and subsequent terms of (3) represent perturbations due to random phase variations in each frequency bin $[36,44]$. To retain the desired unperturbed TRO i.e., $\overline{\mathbf{T}}_{b r}=\overline{\mathbf{K}}_{b r}^{H} \overline{\mathbf{K}}_{b r} \approx \overline{\mathbf{K}}^{H} \overline{\mathbf{K}}=\overline{\mathbf{T}}$ we must completely eliminate or at least minimize the effects of $\overline{\Delta \mathbf{K}}$ i.e., $\overline{\mathbf{T}}_{b r}=\overline{\mathbf{K}}_{b r}^{H} \overline{\mathbf{K}}_{b r} \approx \overline{\mathbf{K}}^{H} \overline{\mathbf{K}}=\overline{\mathbf{T}}$. But in reality, neither $\overline{\mathbf{K}}$ nor $\overline{\Delta \mathbf{K}}$ are known due to the lack of prior knowledge of the target location and the clutter distribution inside a breast of a real patient. To circumvent this, we propose to employ focusing matrices to coherently process the content in each frequency bin to obtain a coherently focused bin in which the perturbation is minimized resulting in a stable TR image. In this, we are guided by the literature on the direction of arrival (DOA) estimation [43, 44] as well as ultrasound imaging [45].

\section{Backpropagation and Imaging}

The backpropagation step in computational TR imaging is carried using a computational medium that must closely resemble the original medium to obtain an accurate image of the target and medium. While designing such a virtual, computational breast medium, one must ensure that its Green's function closely matches with that of the original medium. However, to obtain the Green's function, the actual breast tissue heterogeneity and the dielectric profile of every individual patient is required, which is not easy to estimate. Thus, the degree of mismatch between both the Green's functions sets the limits on the obtainable accuracy of detection and localization when using the TR imaging.

For highly dense breasts, the mismatch can be very high between the original and virtual media Green's functions, which is why many of the existing microwave imaging techniques including TR-MUSIC, DORT and TR-RCB techniques fail to unambiguously resolve even a single, small sized tumor. Hence, we propose a novel TR imaging approach, to obtain superior performance.

When multiple tumors are present inside a highly heterogeneous dense breast medium, the imaging method has to overcome the challenges posed due to (i) the varying scattering strengths owing to the variation in the dielectric contrast, and (ii) the variable path attenuation due to varying distances of travel before arriving at the receiver antenna elements. Our proposed method overcomes these limitations by combining the TR-ML imaging technique with coherent focusing and beamspace processing to obtain higher clutter 
suppression, increased imaging stability, and reduced computational load.

\section{ForWARD PROBLEM SIMULATION}

We have used finite difference time domain (FDTD) [46] simulations employing anatomically realistic, 3-D numerical breast phantoms for the forward problem and obtain the multistatic response data.

\section{A. Computational Test Bed}

The anatomically realistic numerical breast phantoms were obtained from the phantom repository of the University of Wisconsin Cross-Disciplinary Electromagnetics Laboratory (UWCEM) [47]. The phantom repository contains a number of anatomically realistic numerical breast phantoms developed from breast MRI images. These phantoms are mainly used in this paper, to model the interaction between the microwaves and breast tissues realistically and to obtain the multistatic measurement data. A breast phantom along with the transceiver array is illustrated in Fig. 1. The dielectric maps of a highly dense class-4 (C4) breast phantom and a heterogeneously dense class-3 (C3) breast phantom are shown in Fig. 2. The dielectric properties of tumor and different breast tissues have been computed from single pole Debye parameters published in $[5,6]$.

The FDTD method having a grid size of $0.5 \mathrm{~mm}$ in all directions with perfectly matched layer (PML) [46] absorbing boundary is used to calculate the received back scattered responses from the 3-D numerical breast phantoms to form the multistatic matrix. Tumor phantoms with realistic dielectric properties and varying dimensions ranging from $7 \mathrm{~mm}$ to $10 \mathrm{~mm}$ are inserted in the phantoms.

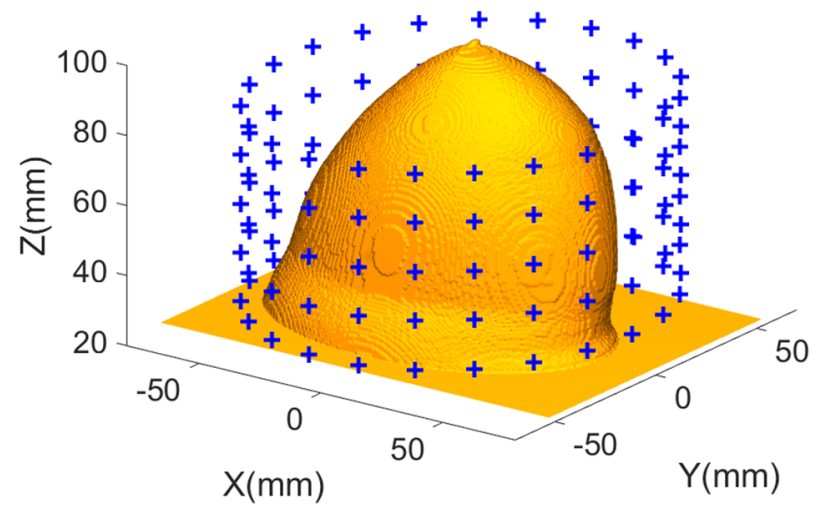

Fig. 2. Highly dense (C4) breast phantom with a cylindrical array.

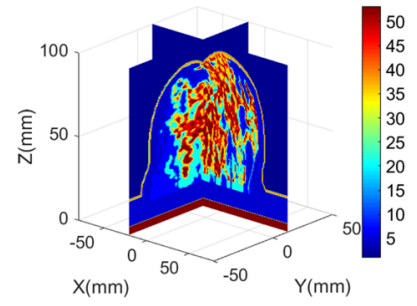

(a)

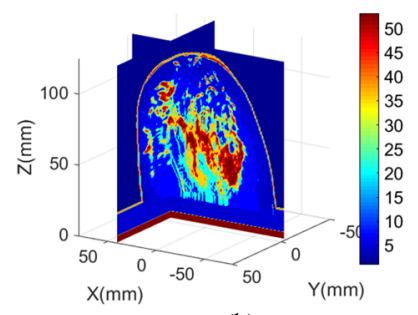

(b)
Fig. 3. Dielectric map of breast phantoms at $2 \mathrm{GHz}$ (a) $\mathrm{C} 4$, and (b) $\mathrm{C} 3$.

The antenna array is formed using ideal Hertzian dipoles which are matched over the entire bandwidth assuming no mutual coupling. A cylindrical transceiver array of size $5 \times 23$ is chosen in which 23 dipole elements are uniformly distributed over each of 5 circular rings that are stacked with equal separation along the vertical $z$-axis. The selected antenna array radius is $70 \mathrm{~mm}$ for the $\mathrm{C} 4$ phantom and $75 \mathrm{~mm}$ for the C3 phantom. The probing microwave UWB signal is a differentiated Gaussian pulse with a 3-dB bandwidth 1.5-5 $\mathrm{GHz}$ with the power spectrum peak occurring at $3 \mathrm{GHz}$. The UWB frequency band is chosen mainly to ensure reasonable resolution while providing sufficient penetration inside breast tissues. The breast phantom along with the array elements is assumed to be immersed in a lossless matching coupling liquid with $\varepsilon_{r}=9$ mainly to avoid excessive skin reflections. The blue markers in Fig. 2 indicate the array element locations.

\section{B. Early time Artifact Removal}

Here, we propose a hybrid technique to remove the skin reflections that form the major part of the early time artifacts. Each antenna positioned at different angular separations as shown in Fig. 2, transmits a probing UWB pulse sequentially into the breast. For each transmission, the backscattered responses from the breast received at all the antennas are recorded to form a multistatic data matrix. The late time arrivals from tissues embedded deep inside the breast are usually much weaker and may potentially contain the signatures of malignant tissues. Hence, to avoid masking of the weak backscattering arising from any malignant lesions, the dominant early-time content must be removed carefully.

The time taken for the backscattered fields to travel from the malignant tissues embedded deep inside the breast and reach the receiver antennas is proportional to the two-way distance from expected tumor location to the antennas and is inversely proportional to the propagation velocity inside the breast medium. Thus, to extract the signatures of malignant lesions, the received late-time signal content must be carefully retained. We propose a wavelet and entropy [48] based hybrid time windowing technique for the early time artifact removal. It operates by first grouping the backscattered skin reflections based on their TOAs and then comparing the entropy levels for each group to detect and remove skin reflections.

During the measurements, the breast is usually made to reside inside an enclosure that also holds the transceiver antenna array. Due to the non-uniform and asymmetric contour of the breast, the distances between the skin and the antenna elements will no longer be the same. Due to this, the skin reflections received at each antenna will have varying time delays. Thus, the design of entropy derived time window is critical for skin artifact removal to prevent any masking of the tumor response. For example, if a malignant lesion is located close to or right beneath the skin, there is a fair chance that its signature gets masked by the strong skin reflections. As a result, this may be unsuspectingly marked for removal as early time artifact, if the entropy derived time window is not selected carefully. To design the time window, first we group the received signals at different antenna elements based on their times of arrival (TOA). The received signals with similar delays and average levels of early time content are grouped so that they approximately fall within the same time window. 
Then, the early time content will be separated for removal by computing entropy for each group.

\section{1) Time of Arrival Estimation}

Once the received signals are grouped, we use complex continuous wavelet transform (CWT) to estimate the TOAs [49] of early time (skin) artifact so as to precisely position the time window obtained from the entropy of the received signal. The CWT of a complex signal is defined as

$$
Q_{s}(a, \tau)=q(\tau) *\left(\frac{1}{\sqrt{a}} \psi_{n}^{*}\left(\frac{\tau}{a}\right)\right)
$$

where, $\psi_{n}(t)=\xi_{n} \frac{d^{n}}{d t^{n}}\left(e^{-j t} e^{-t^{2}}\right)$, and $\xi_{n}$ is a normalization constant so that $\left\|\psi_{n}\right\|^{2}=1$. If the input signal $q(t)$ is scaled and shifted version of $\Psi_{n}$, the coefficient is maximum when $q(t)=h \psi_{n}\left(\frac{t-\tau}{a}\right)$, where $h$ is a scale constant.

To estimate the TOA of the early time skin artifact, we take the wavelet cross spectrum of the excitation signal with the corresponding backscattered response. The delay can be estimated as

$$
\left(\tau_{m}\right)=\arg \max (a, \tau)\left\{\Upsilon\left(Q_{e x c}^{*}(a, \tau) Q_{b s}(a, \tau)\right)\right\}
$$

where, $Q_{e x c}^{*}(a, \tau), Q_{b s}(a, \tau)$ are CWT of the excitation and back scattered response, and $\Upsilon$ denotes smoothing in time and scale.

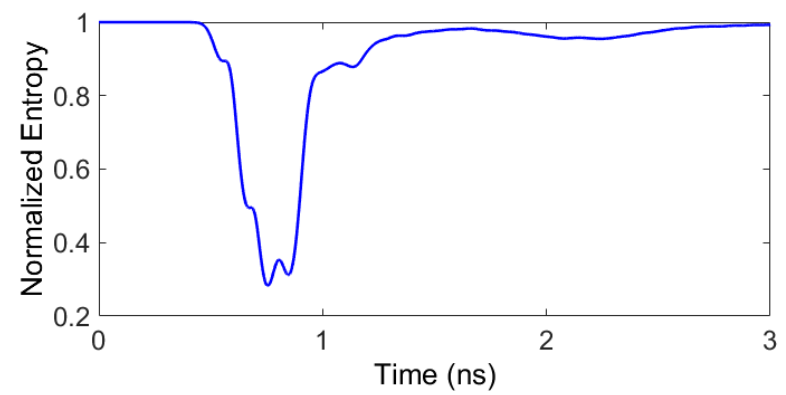

(a)

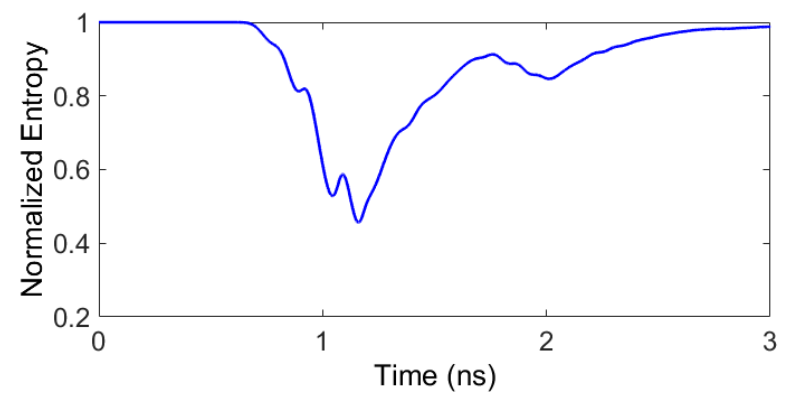

(b)

Fig. 4. Computed entropy of received scattered field for transmit-receive antenna pairs (a) close to the skin, and (b) far from the skin.

\section{2) Entropy window computation}

After grouping the received signals based on their TOAs, the entropy is computed in each group to set-up a threshold for early time artifact removal. For a group of received signals received at $L$ antennas the $\alpha$-th order Rényi entropy [48] can be estimated as

$$
\zeta_{\alpha}(t)=\frac{1}{1-\alpha} \log \left\{\sum_{l=1}^{L}\left[p_{l}(t)\right]^{\alpha}\right\}
$$

where $p_{l}(t)$ is the normalized signal received at $l$-th receiver antenna element and $\alpha$ is a real positive constant. We have used $\alpha=3$. Similar levels of received early time signal content at a group of receiver antennas result in large entropy. Assuming that the early time content is similar to incident fields, we perform entropy smoothing. For a received early time signal, when this smoothed entropy goes beyond a certain pre-determined threshold, then we identify that signal for removal as it could primarily be due to the skin reflections. The computed entropy windows for two different cases are shown in Fig. 4.

\section{Coherent Beamspace Time Reversal}

TR imaging in the time domain suffers from the appearance of ghost target images or false positives due to clutter [39]. Added to this, in low signal to noise ratio (SNR) scenarios, the threshold effect causes the incoherent UWB image to be ineffective [50]. TR imaging in the frequency domain can help to overcome this problem by coherently combining the signal subspaces of individual frequency bins into a full rank coherently focused single frequency bin. Thus, the coherent processing can eliminate spurious peaks resulting from random phase variation in different frequency bins.

\section{A. Coherent Focusing of Time Reversal Operator}

Coherent processing requires derivation of a focusing matrix. A focusing matrix using the wavefield modelling was proposed in [35] for breast cancer detection suitable for TRRCB imaging. However, the wavefield modeling requires spatial interpolation, which could reduce the focusing performance over wide bandwidths particularly, when aiming to detect multiple targets. For this purpose, we derive the coherent focusing matrix by considering the multiple tumors located randomly in the breast at discrete locations. Also, such an approach has been shown to yield lower focusing errors [51]. An autofocusing approach for finding the optimum time instant to obtain a focused image was proposed in [52]. However, in the frequency domain time reversal, it is not necessary to obtain an optimum focusing time instant since the images are reconstructed in the frequency domain. But the signal subspaces in different frequency bins need to be aligned to attain a focused target image.

In highly cluttered media, the eigen values of the TRO rolls off gradually leading to non-unique solutions. When the responses received at different frequency bins are coherently focused, the target response will be enhanced in the resulting focused frequency bin. We propose to use a unitary focusing matrix so that its structure can help to obtain gradual decorrelation of responses from target and clutter. Using the focusing matrix, the signal subspace at each frequency bin can be transformed into a focused subspace spanned by the eigen vectors of the signal subspace in the focused frequency bin. Thus, the focusing matrix $\overline{\mathbf{Z}}[43,53]$ is obtained as

$$
\min _{\overline{\mathbf{Z}}\left(\omega_{f}\right)} E_{\mathbf{r}^{o}}\left\{\left\|\overline{\mathbf{K}}\left(\omega_{o}\right)-\overline{\mathbf{Z}}\left(\omega_{f}\right) \overline{\mathbf{K}}\left(\omega_{f}\right)\right\|_{F}^{2}\right\} \quad \text { subject to } \overline{\mathbf{Z}} \overline{\mathbf{Z}}^{H}=\overline{\mathbf{I}}(7)
$$


Here, $\omega_{0}$ is the focusing frequency where $\omega_{f}(f=1,2, \ldots, F)$ frequency bins are focused, $\|\cdot\|_{F}$ denotes Frobenius norm and $E\{$.$\} denotes expectation. We can expand (7) assuming the$ targets are independent under Born approximation. Considering the probability density function for the targets are expressed as $\rho^{2}(\mathbf{r})=\sum_{p=1}^{P} \wp_{p}(\mathbf{r})$, we can write

$$
\begin{aligned}
& E_{\mathbf{r}^{o}}\left\{\left\|\overline{\mathbf{K}}\left(\omega_{o}\right)-\overline{\mathbf{Z}}^{H}\left(\omega_{f}\right) \overline{\mathbf{K}}\left(\omega_{f}\right)\right\|_{F}^{2}\right\} \\
& \quad=\int_{V} d \mathbf{r}\left\|\rho(\mathbf{r})\left(\overline{\mathbf{K}}\left(\mathbf{r}, \omega_{0}\right)-\overline{\mathbf{Z}}^{H}\left(\omega_{f}\right) \overline{\mathbf{K}}\left(\mathbf{r}, \omega_{f}\right)\right)\right\|_{F}^{2} \\
& \quad=\left\|\overline{\mathbf{K}}\left(\omega_{o}\right)-\overline{\mathbf{Z}}\left(\omega_{f}\right) \overline{\mathbf{K}}\left(\omega_{f}\right)\right\|_{F}^{2}
\end{aligned}
$$

We can now rewrite (8) to get

$$
\begin{aligned}
\min _{\overline{\mathbf{Z}}\left(\omega_{f}\right)}\left\|\overline{\mathbf{K}}\left(\omega_{o}\right)-\overline{\mathbf{Z}}\left(\omega_{f}\right) \overline{\mathbf{K}}\left(\omega_{f}\right)\right\|_{F}^{2} \\
=\operatorname{tr}\left(\overline{\mathbf{K}}^{H}\left(\omega_{o}\right) \overline{\mathbf{K}}\left(\omega_{o}\right)\right)+\operatorname{tr}\left(\overline{\mathbf{K}}^{H}\left(\omega_{f}\right) \overline{\mathbf{K}}\left(\omega_{f}\right)\right) \\
\quad-2 \operatorname{Re}\left\{\operatorname{tr}\left(\overline{\mathbf{K}}\left(\omega_{o}\right) \overline{\mathbf{K}}^{H}\left(\omega_{f}\right) \overline{\mathbf{Z}}^{H}\left(\omega_{f}\right)\right)\right\}
\end{aligned}
$$

The solution of (9) is obtained when $\overline{\mathbf{Z}}=\overline{\mathbf{V}} \overline{\mathbf{U}}^{H}$ where $\overline{\mathbf{K}}^{H}\left(\omega_{f}\right) \overline{\mathbf{K}}\left(\omega_{o}\right)=\overline{\mathbf{U}} \overline{\mathbf{\Sigma}} \overline{\mathbf{V}}^{H}$. Now, we find the coherently focused multistatic matrix as

$$
\overline{\mathbf{K}}_{C}\left(\omega_{o}\right)=\sum_{f=1}^{F} \overline{\mathbf{Z}}\left(\omega_{f}\right) \overline{\mathbf{K}}\left(\omega_{f}\right) \overline{\mathbf{Z}}^{H}\left(\omega_{f}\right)
$$

We can observe the effect of coherent focusing from (10) as

$$
\begin{aligned}
& \overline{\mathbf{Z}}\left(\omega_{f}\right) \overline{\mathbf{K}}\left(\omega_{f}\right) \overline{\mathbf{Z}}^{H}\left(\omega_{f}\right) \\
& =\overline{\mathbf{Z}}\left(\omega_{f}\right) \overline{\mathbf{U}}\left(\omega_{f}\right) \overline{\mathbf{\Phi}}\left(\omega_{f}\right) \overline{\mathbf{V}}^{H}\left(\omega_{f}\right) \overline{\mathbf{Z}}^{H}\left(\omega_{f}\right)=\overline{\mathbf{U}}_{C} \overline{\mathbf{\Phi}}_{C}^{H}
\end{aligned}
$$

The coherent focusing process ensures that, the eigen values in each frequency bin remain unaffected but the eigen vectors are aligned to increase the uniformity of the signal subspace. As the eigen vectors are linked to target locations, the resulting coherently focused TRO will obtain an image that has a brighter focus at the expected target location.

Focusing loss can be used as a metric to measure the quality of the focusing matrix. It is defined as the ratio of array SNR before and after focusing and can be obtained by

$$
\begin{aligned}
& H=\frac{\operatorname{tr}\left\{\overline{\mathcal{K}}_{n}^{-1}\left(\sum_{f=1}^{F} \overline{\mathbf{Z}}_{f} \overline{\mathcal{G}}_{\chi} \overline{\mathcal{G}}^{T} \overline{\mathbf{Z}}_{f}^{H}\right)\right\}}{\operatorname{tr}\left(\sum_{f=1}^{F} \overline{\mathcal{G}} \chi \overline{\mathcal{G}}^{T}\right)} \\
& \overline{\mathcal{K}}_{n}=\frac{\sum_{f=1}^{F} \sigma_{n}^{2}\left(\omega_{f}\right) \overline{\mathbf{Z}}\left(\omega_{f}\right) \overline{\mathbf{Z}}^{H}\left(\omega_{f}\right)}{\sum_{f=1}^{F} \sigma_{n}^{2}\left(\omega_{f}\right)}
\end{aligned}
$$

where, $\sigma_{n}^{2}$ is the noise spectral level and $\overline{\mathcal{K}}_{n}$ is the effective noise covariance after focusing normalized to the noise variance before focusing [43]. For unitary focusing matrix $\overline{\mathbf{Z}}$, we obtain $H=1$. Hence, there is no focusing loss when unitary focusing matrix is employed.

\section{B. Beamspace Processing for Time Reversal Imaging}

Imaging in beamspace is carried out by mapping elementspace data onto beamspace [54]. This offers reduced dimensional processing and additional beamforming gain. Beamspace processing is facilitated by creating a set of orthogonal beams using elementspace data. The space spanned by the output of the beamformers is referred to as the beamspace. Beamspace maximum likelihood estimation was also used for the radar tracking under strong specular multipath [55]. Here, our aim is to combine the coherent focusing with beamspace processing to obtain coherentbeamspace-TR imaging for detecting multiple discrete, tumors in highly dense breast phantoms. The beamspace processing can be carried out after completing the coherent focusing. For beamspace processing, we consider the cylindrical transceiver array that encloses the breast phantom as shown in Fig. 2. We first obtain the beam pattern of the array and then apply beamspace transformation to the focused multistatic matrix given by (10).

\section{1) Beamspace of cylindrical array}

Consider a cylindrical transceiver antenna array as shown in Fig.2 which consists of $C$ concentric circular ring arrays stacked vertically along the z-axis with some finite separation. The array is designed to fully enclose the breast volume. Each circular ring has $D$-antenna elements which are uniformly positioned over its circumference. The vertical separation $Y$ between two consecutive rings is assumed to be $Y<\lambda / 2$. The total number of elements in the cylindrical array is $N=C D$. The antenna elements are all considered to be ideal. Further, it is assumed that every individual antenna element transmits a UWB pulse towards the enclosed breast and the backscattered signals are received at antennas placed at different angular locations surrounding the breast. Hence, the synthesized cylindrical array pattern can be expressed as

$$
A_{c y l}=A_{\text {lin }} \times A_{\text {cir }}
$$

where, $A_{l i n}, A_{c i r}, A_{c y l}$ are the array patterns for a linear array, circular array, and cylindrical array respectively. The beamspace has $m$ modes $m \in[-M, \ldots, 0, \ldots, M]$ and an appropriate number of modes must be chosen in order to minimize the residual error [54].

$$
\mathbf{W}=\left[\begin{array}{ccccc}
1 & \cdots & 1 & \cdots & 1 \\
e^{-j 2 \pi \frac{M}{D}} & \vdots & 1 & \vdots & e^{j 2 \pi \frac{M}{D}} \\
\vdots & \vdots & \vdots & \vdots & \vdots \\
e^{-j 2 \pi M \frac{M(D-1)}{D}} & \vdots & 1 & \vdots & e^{j 2 \pi \frac{M(D-1)}{D}} \\
e^{-j 2 \pi \frac{M}{C}} & \vdots & 1 & \vdots & e^{j 2 \pi \frac{M}{C}} \\
e^{-j 2 \pi\left(\frac{M}{D}+\frac{M}{C}\right)} & \vdots & 1 & \vdots & e^{j 2 \pi\left(\frac{M}{D}+\frac{M}{C}\right)} \\
\vdots & \vdots & \vdots & \vdots & \vdots \\
\vdots & \vdots & \vdots & \vdots & \vdots \\
e^{-j 2 \pi\left(\frac{M(D-1)}{D}+\frac{M(C-1)}{C}\right)} & \cdots & 1 & \cdots & e^{j 2 \pi\left(\frac{M(D-1)}{D}+\frac{M(C-1)}{C}\right)}
\end{array}\right]
$$


The beamspace transformation is obtained using a matrix $\mathbf{W}$ of size $N \times(2 M+1)$ that helps to map elementspace data into beamspace. $\mathbf{W}$ is a matrix that is normalized by the number of array elements $N$. Considering only co-polarized field components of the backscattered signals, matrix $\overline{\mathbf{W}}$ can be written as

$$
\overline{\mathbf{W}}(n, m)=\operatorname{diag}\{\mathbf{W}(n, m), \mathbf{W}(n, m), \mathbf{W}(n, m)\}
$$

where, $\overline{\mathbf{W}}$ is a $3 N \times 3(2 M+1)$ matrix and can be used for beamspace transformation of the dyadic multistatic matrix $\overline{\mathbf{K}}$.

For the beamspace transformation, we choose the number of phase modes for which the Cramer Rao Lower Bound (CRLB) is minimum. The derived CRLB is plotted in Fig. 5 which shows that for $M>5$ where $\mathrm{M}$ denotes the number of phase modes $m=[-M, \ldots . ., M])$, the CRLB is unaffected by the reduced dimensional beamspace transformation due to negligible residual error. The beamspace processing in addition to providing the beamforming gain, reduces the original $3 N \times 3 N$ matrix to $3(2 M+1) \times 3(2 M+1)$, thereby significantly reducing the computational burden. In our case, we have $N=115$ and $M=6$. For deriving the CRLB, we considered a single, small spherical dielectric target embedded in a homogenous dielectric medium. Since the actual breast heterogeneity of any given real patient is unknown, it is difficult to predict the actual Green's function. As a result, the original performance can degrade in heterogeneous breasts compared to the CRLB plotted in Fig. 5 due to homogeneous medium assumption made in its derivation. The CRLB derivation is shown in the appendix.

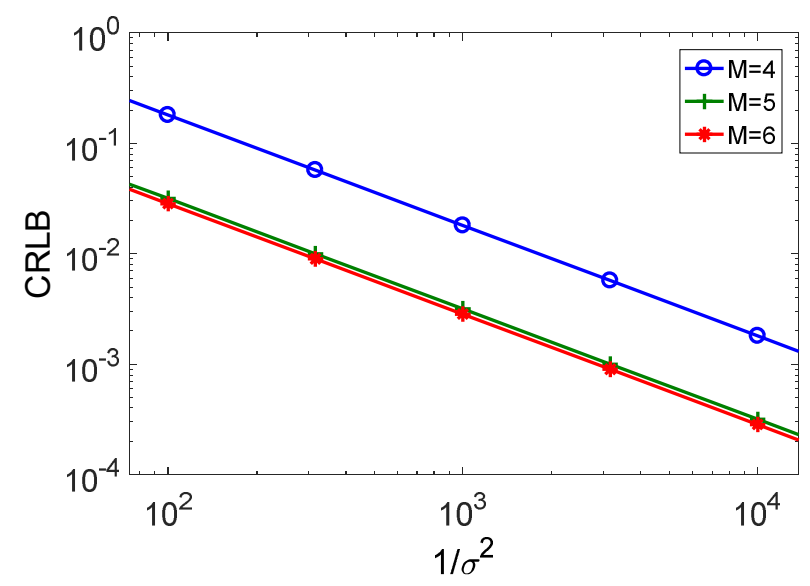

Fig. 5. CRLB for beamspace time reversal imaging.

\section{BACKPROPAGATION AND IMAGING}

In computational TR imaging, as noted earlier, the received signals are first time reversed and then are propagated back into a virtual medium. Usually, the characteristics of the original medium containing the target are hardly known. It was suggested in [39] that use of a computational homogeneous medium whose dielectric constant is equal to the average dielectric constant of the original heterogeneous medium could produce statistically stable TR images. Hence, we consider our computational imaging medium to be a homogeneous medium and attempt to estimate its effective Green's function to carry out TR imaging.
For computing the Green's function, we must first estimate the dielectric properties of the virtual medium. For microwave imaging in general, the average dielectric property estimation is important $[20,56]$. Here, we propose a phantom specific TOA based technique for the estimation of the average dielectric constant of the breast medium. In the proposed technique, we first form many transmit-receive antenna pairs by positioning them on opposite sides of the breast i.e., approximately $180^{\circ}$ apart in the azimuthal direction. The aim is to ensure that a pulse radiated by one antenna of the pair will travel through complete width of the breast before its pair antenna located at the opposite end receives it. We then use the signals transmitted through breast tissue to estimate the TOAs from which the average dielectric constant of an equivalent, homogeneous virtual imaging medium is calculated by minimizing the following functional

$$
\min _{\varepsilon} \sum_{i}\left(\frac{t_{i}^{2}}{d_{i}^{2} \mu_{0} \varepsilon_{0}}-\varepsilon\right)
$$

where, $d_{i}$ is the distance between $i$-th transmitter-receiver pair and $t_{i}$ is the associated TOA, $\varepsilon$ is the dielectric constant of the virtual homogeneous computational medium, $\mu_{0}$ and $\varepsilon_{0}$ are the permeability and permittivity of free space respectively. Our investigations have revealed that the signals from antennas positioned in three central circular rings of the cylindrical array (Fig. 2) are sufficient to estimate the average dielectric constant of the virtual homogeneous medium. The estimated dielectric constant is then used to compute an effective Green's function for TR imaging.

TR image is a bright focus at the expected location of the target. However, a gradual increase in the mismatch between Green's functions of computational and actual breast media leads to loss of energy intensity at the focus region. A high intensity TR focus that is formed at the desired location can be used as an indication of minimal Green's function mismatch in the absence of the knowledge of the actual breast tissue composition of a real patient. The proposed technique can offer reasonable compensation suitable for practical applications.

\section{A. Coherent Beamspace Time Reversal Maximum Likelihood Imaging}

The time reversal maximum likelihood (TR-ML) method when used in elementspace can be computationally exhaustive as it involves numerical search within full 3-D breast volume. To reduce the computational load as well as to achieve beamforming gain coupled with advantages of coherent focusing, we propose to combine the beamspace processing with the coherently focused TRO to realize C-B-TR-ML imaging. The beamspace processing is applied in both transmit and receive modes. Thus, the coherently focused beamspace multistatic matrix can be expressed as

$$
\begin{aligned}
\overline{\mathbf{K}}_{C B} & =\overline{\mathbf{W}}^{H} \overline{\mathbf{K}}_{C} \overline{\mathbf{W}}=\overline{\mathbf{W}}^{H} \overline{\mathcal{G}}_{\bar{\chi}}\left(\overline{\mathbf{W}}^{T} \overline{\mathcal{G}}\right)^{T}+\sigma^{2} \overline{\mathbf{W}}^{H} \overline{\mathbf{I} \mathbf{W}} \\
& =\overline{\mathcal{G}}_{B_{r}} \bar{\chi} \overline{\mathcal{G}}_{B_{t}}^{T}+\sigma^{2} \overline{\mathbf{I}}_{B} \\
& =\overline{\mathbf{K}}_{C B}+\overline{\boldsymbol{\eta}}_{B}
\end{aligned}
$$


where, $\overline{\mathbf{I}}_{B}$ is a $(2 M+1) \times(2 M+1)$ unit dyad. Considering the fact that, $N>(2 M+1), \overline{\mathbf{K}}_{C B}$ results in a reduced dimension of $3(2 M+1) \times 3(2 M+1)$. Even in beamspace domain, the noise remains zero mean and Gaussian distributed. Hence the likelihood function for the location and strengths of the scatterers (targets) can be expressed as [38]

$$
l\left(\mathbf{r}, \bar{\chi}, \sigma^{2} ; \overline{\mathbf{K}}_{C B}\right)=\frac{1}{\left(\pi \sigma^{2}\right)^{N^{2}}} \exp \frac{-\|\|_{C B}-\left.\overline{\mathbf{k}}_{C B}(\mathbf{r}, \bar{\chi})\right|_{F} ^{2}}{\sigma^{2}}
$$

As stated earlier, we consider only the co-polar scattered field components for processing the maximum likelihood estimate of $\bar{\chi}$ which can be obtained as

$$
\begin{aligned}
\overline{\tilde{\chi}}, \tilde{\mathbf{r}} & =\arg \min \left\|\overline{\mathbf{K}}_{C B}-\overline{\mathbf{K}}_{C B}(\mathbf{r}, \overline{\boldsymbol{\chi}})\right\|_{F}^{2} \\
& =\arg \min \left\|\operatorname{vec}\left(\overline{\mathbf{K}}_{C B}\right)-\left(\overline{\mathcal{G}}_{B_{t}} \odot \overline{\mathcal{G}}_{B_{r}}\right) \operatorname{vec}(\bar{\chi})\right\|_{F}^{2}
\end{aligned}
$$

Where, $\odot$ denotes Khatri-Rao product [38]. The dielectric tensor can be estimated using the least square solution at each voxel within the breast volume as

$$
\overline{\tilde{\chi}}(\mathbf{r})=\left[\overline{\mathcal{G}}_{B \odot}^{H}(\mathbf{r}) \overline{\mathcal{G}}_{B \odot}(\mathbf{r})\right]^{-1} \overline{\mathcal{G}}_{B \odot}^{H}(\mathbf{r}) \operatorname{vec}\left(\overline{\mathbf{K}}_{C B}\right)
$$

where, $\overline{\mathcal{G}}_{B \odot}^{H}=\overline{\mathcal{G}}_{B_{t}} \odot \overline{\mathcal{G}}_{B_{r}}$. For imaging purposes, the whole breast volume is divided into a large number of small voxels and each voxel is searched for the presence of the potential targets based on their scattering field strengths. The imaging metric for the proposed C-B-TR-ML method is given by

$$
I_{C-B-T R-M L}=\frac{1}{\left\|P_{\overline{\mathbf{g}}_{B \otimes}}^{\perp} \operatorname{vec}\left(\overline{\mathbf{K}}_{C B}\right)\right\|_{F}^{2}}=\left\|P_{\overline{\mathbf{g}}_{B \otimes}} \operatorname{vec}\left(\overline{\mathbf{K}}_{C B}\right)\right\|_{F}^{2}
$$

where, $\overline{\mathbf{K}}_{C B}$ is obtained by coherently focusing $\overline{\mathbf{K}}$ prior to beamspace imaging, $P_{\overline{\mathbf{g}}_{B \otimes}}^{\perp}=\overline{\mathbf{I}}-P_{\overline{\mathbf{g}}_{B \otimes}}=\overline{\mathbf{I}}-\overline{\mathbf{g}}_{B \otimes}\left[\overline{\mathbf{g}}_{B \otimes} \overline{\mathbf{g}}_{B \otimes}\right]^{-1} \overline{\mathbf{g}}_{B \otimes}^{H}$, and $\overline{\mathbf{g}}_{B \otimes}(\mathbf{r})=\overline{\mathbf{g}}_{B_{t}}(\mathbf{r}) \otimes \overline{\mathbf{g}}_{B_{r}}(\mathbf{r})$.

\section{B. Coherent Beamspace Time Reversal Subspace Imaging}

Beamspace processing and coherent focusing preserve the subspace orthogonality. Hence, subspace based techniques such as TR-MUSIC and DORT can also benefit from coherent beamspace processing. Through the eigen decomposition of C-B-TRO, we can obtain the orthogonal signal and noise subspaces. DORT imaging is carried out with the signal subspace while TR-MUSIC imaging requires the noise subspace. The eigen values and corresponding eigen vectors are obtained from the eigen decomposition of $\overline{\mathbf{T}}_{C B}$ which can be represented as

$$
\overline{\mathbf{T}}_{C B}={\overline{\mathbf{V}_{\mathbb{S}}^{C B}}}_{\mathbf{\Phi}_{\mathbb{S}}^{C B}}^{2}{\overline{\mathbf{V}_{\mathbb{S}}^{C B}}}^{H}+{\overline{\mathbf{V}_{\mathbb{N}}^{C B}}}_{\mathbf{\Phi}_{\mathbb{N}}^{C B}}^{2}{\overline{\mathbf{V}_{\mathbb{N}}^{C B}}}^{H}
$$

The eigen decomposition of the C-B-TRO yields its eigen vectors and eigen values. The signal subspace is spanned by $\overline{\mathbf{V}_{\mathbb{S}}^{C B}}$ and $\overline{\boldsymbol{\Phi}_{\mathbb{S}}^{C B}}$ includes the significant singular values $\left(\overline{\Phi_{n}^{C B}}>0, n=1,2, \ldots, s\right)$. Similarly, the noise subspace, $\mathbf{V}_{\mathbb{N}}^{C B}$ corresponding to the insignificant singular values is also obtained as $\left(\overline{\boldsymbol{\Phi}_{n}^{C B}} \approx 0, n=s+1, s+2, \ldots, 2 M+1\right)$. The Green's function vectors of the background medium form the orthonormal bases of the signal subspace [57]. We use Akaike Information Criterion (AIC) to estimate the two orthogonal subspaces [9]. Once the signal and noise subspaces are estimated using AIC, we use the following imaging functions.

$$
\begin{aligned}
& I_{C-B-D O R T}(\mathbf{x})=\sum_{n=1}^{s_{A C}}\left\|{\overline{\mathbf{v}_{n}^{C B}}}^{H} \overline{\mathbf{g}}_{B}(\mathbf{x})\right\|_{F}^{2} \\
& I_{C-B-T R-M U S I C}(\mathbf{x})=\frac{1}{\sum_{n=s_{A I C}+1}^{2 M+1}\left\|{\overline{\mathbf{v}_{n}^{C B}}}^{H} \overline{\mathbf{g}}_{B}(\mathbf{x})\right\|_{F}^{2}}
\end{aligned}
$$

\section{RESUlTS}

In order to demonstrate the capabilities of the proposed coherent beamspace TR microwave imaging techniques, we investigate the detection of multiple tumors inserted into $\mathrm{C} 4$ and $\mathrm{C} 3$ breast phantoms. Firstly, we consider two $10 \mathrm{~mm}$ sized tumors in a highly dense $\mathrm{C} 4$ breast phantom as shown in Fig. 6. The tumors are inserted approximately at 2 o'clock and 4 o'clock positions respectively from an enface view and at a depth of $4.5 \mathrm{~cm}$ under the nipple. The image obtained by the C-B-TR-MUSIC, as shown in Fig. 6(a), reveals two high intensity regions indicating possible presence of tumors at those locations. The location accuracy for a tumor at 2 o'clock position is quite satisfactory but the other at 4 o'clock position is not accurately localized as the bright spot is formed away from the actual tumor locations as indicated in Fig. 6 using spherical inclusions. Now, we consider the result obtained by C-B-DORT method shown in Fig. 6(b). The figure indicates that both the tumors are accurately identified although there appears additional high intensity region formed away from the tumor locations. In both these images, one can observe significant levels of energy being dispersed in sidelobes. On the other hand, observe the result shown in Fig. 6(c) which is obtained by the C-B-TR-ML method. The C-B-TR-ML imaging offers by far the best image in which both the tumors are accurately localized without significant sidelobes. However, the estimated location of the tumor positioned at 2 o'clock is slightly shifted above its true location as can be seen in Fig. 6(c).

Next, consider the images of Fig. 7 concerning the detection of two $10 \mathrm{~mm}$ size tumors inserted at angular positions 12:30 o'clock and 7 o'clock and at depths of $6 \mathrm{~cm}$ and $8.5 \mathrm{~cm}$ beneath the nipple respectively in a $\mathrm{C} 3$ phantom. It can be noticed from Fig. 7(a) that the image produced by the C-BTR-MUSIC technique displays high intensity spots both closer to as well as far away from actual tumor locations. In contrast, images in Fig. 7(b) and Fig. 7(c) produced by C-B-DORT and C-B-TR-ML techniques respectively, show high intensity spots only at the true tumor locations.

We now consider a challenging imaging scenario involving a highly dense $\mathrm{C} 4$ breast phantom in which two, small sized, $7 \mathrm{~mm}$ diameter tumors are inserted, one at 1 o'clock position at a depth of $2 \mathrm{~cm}$ below nipple and the other inserted at 10:30 o'clock position at a depth of $6.5 \mathrm{~cm}$ below nipple. The image shown in Fig. 8 (c) that is obtained by the C-B-TR-ML technique shows high intensity foci near the true tumor 
locations with relatively low sidelobe dispersion. This reconfirms the capabilities of the proposed C-B-TR-ML approach for the detection of multiple, small sized tumors. The images in Fig. 8(a)-(b) show the results obtained by C-B-TRMUSIC and C-B-DORT techniques for two $7 \mathrm{~mm}$ tumors in the $\mathrm{C} 4$ breast phantom. Although some side lobes are present, the tumor locations can still be identified from the focus region since the high intensity regions are formed very close to the actual tumor locations. This can be mainly attributable to the use of proposed coherent beamspace TR imaging technique. However, the resolution and accuracy achieved in images obtained by the C-B-TR-ML technique, clearly appears to be superior to those computed using C-B-DORT and C-B-TR-MUSIC methods.

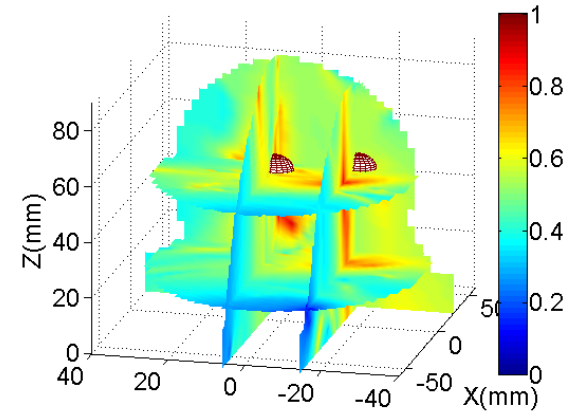

(a)

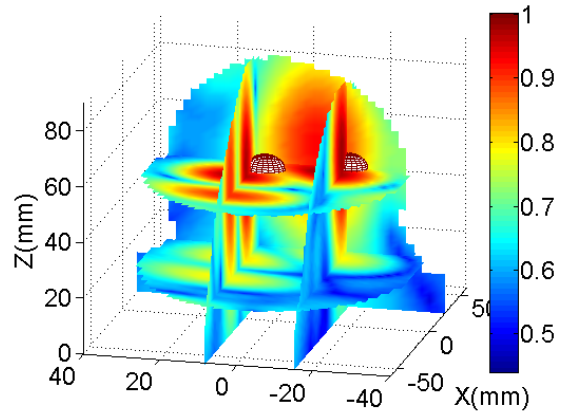

(b)

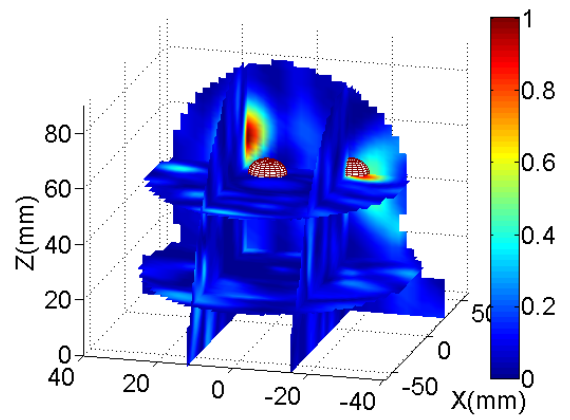

(c)

Fig. 6. Detection of two $10 \mathrm{~mm}$ size tumors embedded inside a highly dense (C4) phantom: (a) C-B-TR-MUSIC, (b) C-B-DORT, and (c) C-B-TR-ML.

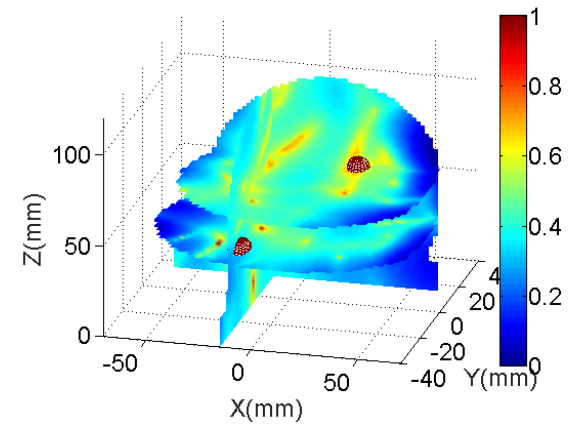

(a)

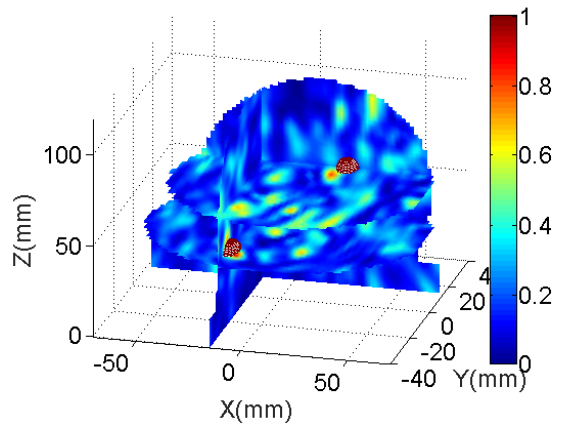

(b)

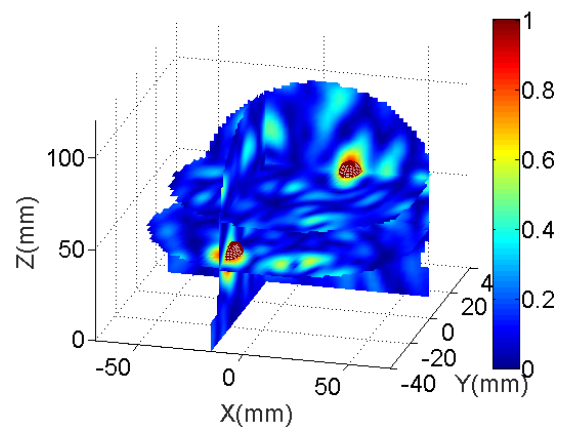

(c)

Fig. 7. Detection of two $10 \mathrm{~mm}$ size tumors embedded inside a heterogeneously dense (C3) phantom: (a) C-B-TR-MUSIC, (b) C-B-DORT, and (c) C-B-TR-ML.

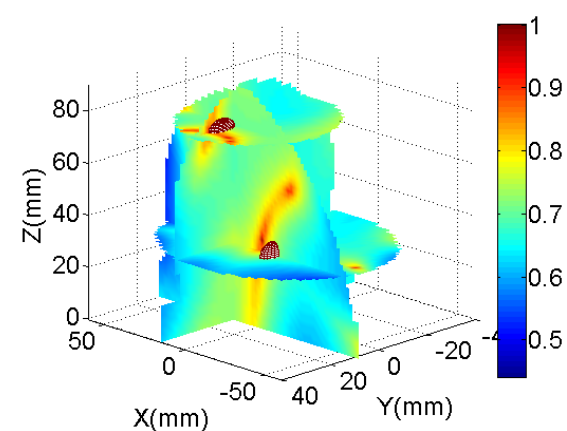

(a)

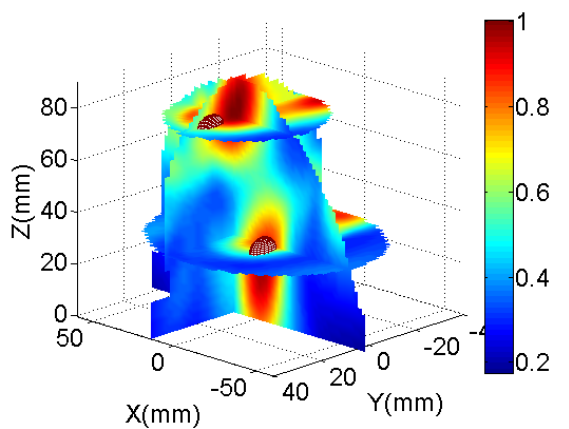

(b)

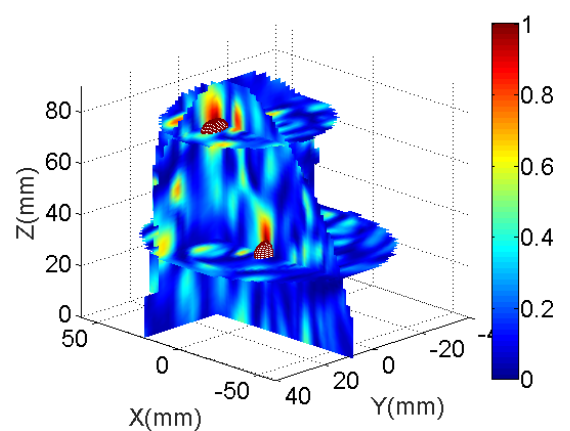

(c)

Fig. 8. Detection of two $7 \mathrm{~mm}$ sized tumors embedded inside a highly dense (C4) phantom: (a) C-B-TR-MUSIC, (b) C-B-DORT, and (c) C-B-TR-ML.

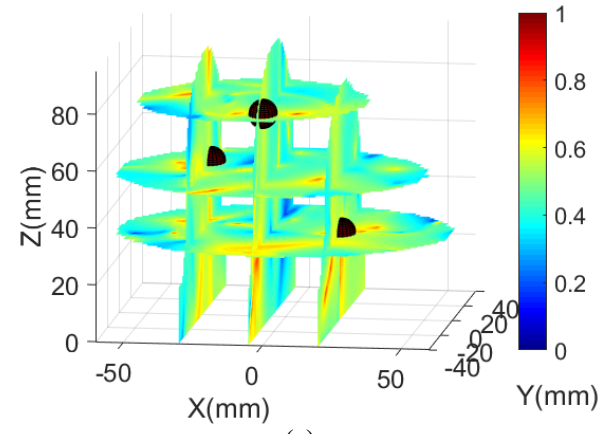

(a)

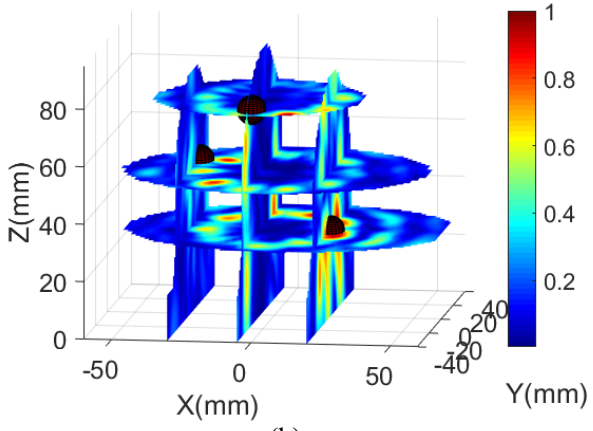

(b)

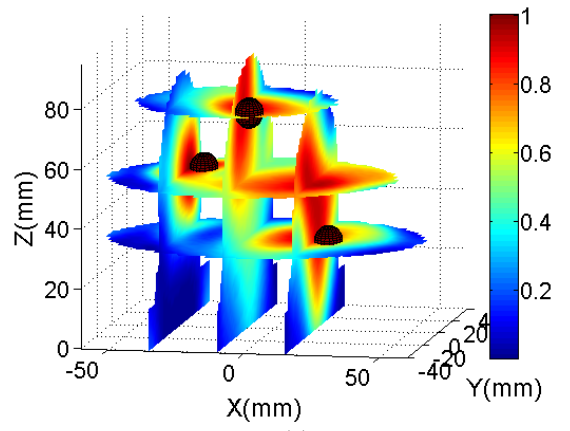

(c)

Fig. 9. Comparison of the detection performance of three $10 \mathrm{~mm}$ sized tumors inside a C4 phantom: (a) C-B-TR-MUSIC, (b) C-B-DORT, and (c) C-B-TR-ML. 
Finally, we consider another highly challenging scenario where three $10 \mathrm{~mm}$ sized spherical shaped tumors are inserted in a highly dense $\mathrm{C} 4$ breast phantom. The three tumors are located at 6 o'clock position at a depth of $2 \mathrm{~cm}$ below the nipple, 10 o'clock position at a depth of $4.5 \mathrm{~cm}$ below the nipple and the third at 3 o'clock position at a depth of $6.5 \mathrm{~cm}$ below the nipple, respectively. The C-B-TR-ML image shown in Fig. 9(c) reveals that there are high intensity foci formed close to all the three tumor locations. However, unlike the previous examples, high intensity energy peaks are spread over larger regions within the breast. The results shown in Fig. 9(a)-(b) which are obtained by C-B-TR-MUSIC and C-BDORT methods for the same three tumor case, evidently fail to unambiguously localize them. This, once again, demonstrates the superior capabilities of the proposed C-BTR-ML approach for the detection of multiple tumors in a highly dense, $\mathrm{C} 4$ breast phantom.

To investigate the role played by coherent processing, we tested it by removing the coherent focusing and used only BTR-ML to find that all the three tumor locations could not be unambiguously estimated. Thus, the role of coherent processing appears to be crucial for successfully locating all the three tumors in highly dense, $\mathrm{C} 4$ phantoms. We have obtained Figs. 6-9 at $\mathrm{SNR}=40 \mathrm{~dB}$ where the noise was added to the received scattered field responses.

In order to further illustrate the advantages of proposed CB-TR-ML imaging, we compute peak to side lobe ratio (PSLR) for the results shown in Figs. 6-9 and tabulated in Table I.

TABLE I

PSLR FOR DIFFERENT CASES OF TUMOR DETECTION

\begin{tabular}{|c|c|c|c|}
\hline Case & $\begin{array}{l}\text { Tumor Position } \\
\text { (o'clock, Depth) }\end{array}$ & TR Technique & $\begin{array}{c}\text { PSLR } \\
(\mathrm{dB})\end{array}$ \\
\hline \multirow{6}{*}{$\begin{array}{l}\text { Two } 10 \mathrm{~mm} \\
\text { tumors in C4 } \\
\text { Phantom }\end{array}$} & 2 o'clock, $4.5 \mathrm{~cm}$ & C-B-DORT & 0.56 \\
\hline & 4 o'clock, $4.5 \mathrm{~cm}$ & C-B-DORT & 0.66 \\
\hline & 2 o'clock, $4.5 \mathrm{~cm}$ & C-B-TR-MUSIC & 0.47 \\
\hline & 4 o'clock, $4.5 \mathrm{~cm}$ & C-B-TR-MUSIC & 0.56 \\
\hline & 2 o'clock, $4.5 \mathrm{~cm}$ & C-B-TR-ML & 3.22 \\
\hline & 4 o'clock, $4.5 \mathrm{~cm}$ & C-B-TR-ML & 2.04 \\
\hline \multirow{6}{*}{$\begin{array}{l}\text { Two } 7 \mathrm{~mm} \text { tumors } \\
\text { in C4 Phantom }\end{array}$} & 1 o'clock, $2 \mathrm{~cm}$ & C-B-DORT & 0.90 \\
\hline & $10: 30$ o'clock, $6.5 \mathrm{~cm}$ & C-B-DORT & 1.08 \\
\hline & 1 o'clock, $2 \mathrm{~cm}$ & C-B-TR-MUSIC & 0.36 \\
\hline & $10: 30$ o'clock, $6.5 \mathrm{~cm}$ & C-B-TR-MUSIC & 0.58 \\
\hline & 1 o'clock, $2 \mathrm{~cm}$ & C-B-TR-ML & 2.19 \\
\hline & $10: 30$ o'clock, $6.5 \mathrm{~cm}$ & C-B-TR-ML & 2.62 \\
\hline \multirow{3}{*}{$\begin{array}{c}\text { Three } 10 \mathrm{~mm} \\
\text { tumors in } \mathrm{C} 4 \\
\text { Phantom }\end{array}$} & 6 o'clock, $2 \mathrm{~cm}$ & C-B-TR-ML & 2.31 \\
\hline & 10 o'clock, $4.5 \mathrm{~cm}$ & C-B-TR-ML & 2.63 \\
\hline & 3 o'clock, $6.5 \mathrm{~cm}$ & C-B-TR-ML & 3.74 \\
\hline \multirow{6}{*}{$\begin{array}{l}\text { Two } 10 \mathrm{~mm} \\
\text { tumors in C3 } \\
\text { Phantom }\end{array}$} & 12:30 o'clock, $6 \mathrm{~cm}$ & C-B-DORT & 0.72 \\
\hline & 7 o'clock, $8.5 \mathrm{~cm}$ & C-B-DORT & 1.43 \\
\hline & $12: 30$ o'clock, $6 \mathrm{~cm}$ & C-B-TR-MUSIC & -0.48 \\
\hline & 7 o'clock, $8.5 \mathrm{~cm}$ & C-B-TR-MUSIC & 1.42 \\
\hline & $12: 30$ o'clock, $6 \mathrm{~cm}$ & C-B-TR-ML & 1.11 \\
\hline & 7 o'clock, $8.5 \mathrm{~cm}$ & C-B-TR-ML & 2.07 \\
\hline
\end{tabular}

\section{CONCLUSION}

We have proposed the coherent beamspace maximum likelihood method for TR imaging. Also, the coherent, beamspace approach has been extended for TR-MUSIC and DORT algorithms. We have demonstrated that all three proposed imaging techniques offer robust performance in highly dense breasts. In particular, it has been verified that the proposed C-B-TR-ML technique performs better than C-BTR-MUSIC and C-B-DORT for accurately resolving multiple tumors in highly dense breast phantoms. We have derived a unitary coherent focusing matrix that has zero focusing loss. The beamspace transformation derived for a 3-D cylindrical array has offered significant reduction in computational burden. A hybrid technique has also been proposed to accurately remove the early time skin artifacts.

We have also proposed a phantom specific approach to estimate the Green's function of an equivalent virtual homogeneous medium for TR operation. It must be recognized that increased dielectric mismatch between the virtual and original breast media can adversely affect the imaging quality. Our work demonstrates that C-B-TR-ML microwave imaging is effective for detecting multiple tumors in highly dense breasts, where other existing imaging techniques tend to fail. It has also been substantiated that the proposed beamspace processing has greatly reduced the computational burden since ML estimation is carried out only on a $39 \times 39$ matrix instead of the original $445 \times 445$ elementspace multistatic matrix for the 115-element antenna array. As a result, UWB image can be obtained within minutes. It can be concluded that the proposed C-B-TR-ML method can offer further enhancement of microwave imaging so that it can become an attractive alternative/complementary low-cost imaging modality for early stage breast cancer screening.

\section{APPENDIX I}

Cramer Rao Lower Bound (CRLB) is known to provide fundamental limit on estimation accuracy. CRLB for multistatic point scatterer estimation was formulated in [58]. Maximum likelihood estimation of scatterer location $\mathbf{r}$ can be computed from (20). We define $\overline{\boldsymbol{\theta}}=\left[\overline{\tilde{\tau}}^{T}, \tilde{\mathbf{r}}^{T}\right]$ to find the Fisher Information Matrix (FIM) as

$$
\begin{aligned}
& \overline{\mathcal{I}}(\overline{\boldsymbol{\theta}})=-E\left\{\frac{\partial^{2} l\left(\mathbf{r}, \overline{\boldsymbol{\tau}}, \sigma^{2} ; \overline{\mathbf{K}}_{B}\right)}{\partial \overline{\boldsymbol{\theta}} \partial \overline{\boldsymbol{\theta}}^{T}}\right\} \\
& =\frac{2}{\sigma^{2}} \mathfrak{R}\left\{\left[\frac{\partial v e c\left(\overline{\mathbf{K}}_{B}(\mathbf{r}, \overline{\boldsymbol{\tau}})\right)}{\partial \overline{\boldsymbol{\theta}}^{T}}\right]^{H}\left[\frac{\partial v e c\left(\overline{\mathbf{K}}_{B}(\mathbf{r}, \overline{\boldsymbol{\tau}})\right)}{\partial \overline{\boldsymbol{\theta}}^{T}}\right]\right\} \\
& =\frac{2}{\sigma^{2}} \mathfrak{R}\left\{\overline{\mathbf{S}}^{H}(\overline{\boldsymbol{\theta}}) \overline{\mathbf{S}}(\overline{\boldsymbol{\theta}})\right\}
\end{aligned}
$$

Here,

$$
\begin{gathered}
\overline{\mathbf{S}}(\overline{\boldsymbol{\theta}})=\frac{\partial \operatorname{vec}\left(\overline{\mathbf{K}}_{B}(\mathbf{r}, \overline{\boldsymbol{\tau}})\right)}{\partial \overline{\boldsymbol{\theta}}^{T}}=\left[\overline{\mathbf{S}}_{\overline{\boldsymbol{\tau}}} \overline{\mathbf{S}}_{\mathbf{r}}\right] \\
\overline{\mathbf{S}}_{\bar{\tau}}=\frac{\partial \operatorname{vec}\left(\overline{\mathbf{K}}_{B}(\mathbf{r}, \overline{\boldsymbol{\tau}})\right)}{\partial \overline{\boldsymbol{\tau}}^{T}}
\end{gathered}
$$




$$
\overline{\mathbf{S}}_{\mathbf{r}}=\frac{\partial v e c\left(\overline{\mathbf{K}}_{B}(\mathbf{r}, \overline{\boldsymbol{\tau}})\right)}{\partial \mathbf{r}^{T}}
$$

Hence, the FIM can be expressed as

$$
\overline{\mathcal{I}}(\overline{\boldsymbol{\theta}})=\left[\begin{array}{ll}
\overline{\boldsymbol{I}}_{\bar{\tau} \bar{\tau}} & \overline{\boldsymbol{I}}_{\mathbf{r} \bar{\tau}}^{T} \\
\overline{\mathcal{I}}_{\mathbf{r} \bar{\tau}} & \overline{\boldsymbol{I}}_{\mathbf{r r}}
\end{array}\right]
$$

Thus, the CRLB is obtained as

$$
\begin{aligned}
\operatorname{CRLB}(\overline{\boldsymbol{\theta}}) & =\left[\begin{array}{ll}
\overline{\mathcal{I}}_{\overline{\bar{\tau}} \bar{\tau}} & \overline{\boldsymbol{I}}_{\mathbf{r} \bar{\tau}}^{T} \\
\overline{\mathcal{I}}_{\mathbf{r} \bar{\tau}} & \overline{\boldsymbol{I}}_{\mathrm{rr}}
\end{array}\right]^{-1} \\
& =\left[\begin{array}{ll}
\mathrm{CRB}_{\bar{\tau} \bar{\tau}} & \mathrm{CRB}_{\mathrm{r} \bar{\tau}}^{T} \\
\mathrm{CRB}_{\overline{\mathrm{r}} \bar{\tau}} & \mathrm{CRB}_{\mathrm{rr}}
\end{array}\right]
\end{aligned}
$$

Eventually, the CRLB for location estimation is attained as

$$
C R L B_{\mathrm{rr}}=\left(\overline{\boldsymbol{I}}_{\mathrm{rr}}-\overline{\boldsymbol{I}}_{\mathrm{r} \boldsymbol{\tau}} \overline{\boldsymbol{I}}_{\bar{\tau} \bar{\tau}} \overline{\boldsymbol{I}}_{\mathrm{r} \bar{\tau}}\right)^{-1}
$$

We have assumed free space Green's function for FIM computation to get $\frac{\partial G}{\partial R}=\frac{\partial\left(e^{j k R} / 4 \pi R\right)}{\partial R}=\frac{e^{j k R}(j k R-1)}{4 \pi R^{2}}$.

\section{REFERENCES}

[1] Cancer Council Australia. Available: http://www.cancer.org.au/ ABOUT-CANCER/EARLY-DETECTION/EARLY-DETECTIONFACTSHEETS/BREAST-CANCER.HTML

[2] D. W. Winters, J. D. Shea, P. Kosmas, B. D. Van Veen, and S. C. Hagness, "Three-Dimensional Microwave Breast Imaging: Dispersive Dielectric Properties Estimation Using Patient-Specific Basis Functions," IEEE Trans. Med. Imag., vol. 28, pp. 969-981, 2009.

[3] W. A. Berg, L. Gutierrez, M. S. NessAiver, W. B. Carter, M. Bhargavan, R. S. Lewis, et al., "Diagnostic Accuracy of Mammography, Clinical Examination, US, and MR Imaging in Preoperative Assessment of Breast Cancer," Radiology, vol. 233, pp. 830-849, 2004.

[4] A. M. Hassan and M. El-Shenawee, "Review of Electromagnetic Techniques for Breast Cancer Detection," IEEE Reviews Biomed. Eng., vol. 4, pp. 103-118, 2011.

[5] M. Lazebnik, D. Popovic, L. McCartney, C. B. Watkins, M. a. J. Lindstrom, J. Harter, et al., "A large-scale study of the ultrawideband microwave dielectric properties of normal, benign and malignant breast tissues obtained from cancer surgeries " Phys. Med. Biol., vol. 52, pp. 6093-6115, 2007.

[6] M. Lazebnik, M. Okoniewski, J. H. Booske, and S. C. Hagness, "Highly accurate debye models for normal and malignant breast tissue dielectric properties at microwave frequencies," IEEE Microw. Wireless Comp. Lett., vol. 17, pp. 822-824, 2007.

[7] H. F. Abutarboush and M. Klemm, "Signal Selection for ContrastEnhanced UWB Microwave Radar Imaging With Inhomogeneous Breast Phantoms," IEEE Antennas Wireless Propag. Lett., vol. 12, pp. 1408$1411,2013$.

[8] E. Kirshin, B. Oreshkin, G. K. Zhu, M. Popovic, and M. Coates, "Microwave Radar and Microwave-Induced Thermoacoustics: DualModality Approach for Breast Cancer Detection," IEEE Trans. Biomed. Eng., vol. 60, pp. 354-360, 2013.

[9] Y. Chen and P. Kosmas, "Detection and Localization of Tissue Malignancy Using Contrast-Enhanced Microwave Imaging: Exploring Information Theoretic Criteria," IEEE Trans. Biomed. Eng., vol. 59, pp. 766-776, 2012

[10] M. Guardiola, S. Capdevila, J. Romeu, and L. Jofre, "3-D Microwave Magnitude Combined Tomography for Breast Cancer Detection Using Realistic Breast Models," IEEE Antennas Wirel. Propag. Lett., vol. 11, pp. 1622-1625, 2012.

[11] J. D. Shea, B. D. Van Veen, and S. C. Hagness, "A TSVD Analysis of Microwave Inverse Scattering for Breast Imaging," IEEE Trans. Biomed. Eng., vol. 59, pp. 936-945, 2012.

[12] T. M. Grzegorczyk, P. M. Meaney, P. A. Kaufman, R. M. di FlorioAlexander, and K. D. Paulsen, "Fast 3-D Tomographic Microwave Imaging for Breast Cancer Detection," IEEE Trans. Med. Imag., vol. 31, pp. $1584-1592,2012$
[13] M. El-Shenawee and E. L. Miller, "Spherical harmonics microwave algorithm for shape and location reconstruction of breast cancer tumor," IEEE Trans. Med. Imag., vol. 25, pp. 1258-1271, 2006.

[14] A. E. Fouda and F. L. Teixeira, "Ultra-wideband microwave imaging of breast cancer tumors via Bayesian inverse scattering," J. Appl. Phys., vol. 115, p. 064701, 2014.

[15] M. Klemm, I. J. Craddock, J. A. Leendertz, A. Preece, and R. Benjamin, "Radar-Based Breast Cancer Detection Using a Hemispherical Antenna Array-Experimental Results," IEEE Trans. Antennas Propag., vol. 57, pp. 1692-1704, 2009.

[16] M. O'Halloran, E. Jones, and M. Glavin, "Quasi-Multistatic MIST Beamforming for the Early Detection of Breast Cancer," IEEE Trans. Biomed. Eng., vol. 57, pp. 830-840, 2010.

[17] E. C. Fear, J. Bourqui, C. Curtis, D. Mew, B. Docktor, and C. Romano, "Microwave Breast Imaging With a Monostatic Radar-Based System: A Study of Application to Patients," IEEE Trans. Microw. Theory Tech., vol. 61, pp. 2119-2128, 2013.

[18] E. Porter, E. Kirshin, A. Santorelli, M. Coates, and M. Popovic, "TimeDomain Multistatic Radar System for Microwave Breast Screening," IEEE Antennas Wireless Propag. Lett., vol. 12, pp. 229-232, 2013.

[19] E. Porter, M. Coates, and M. Popović, "An Early Clinical Study of Time-Domain Microwave Radar for Breast Health Monitoring," IEEE Trans. Biomed. Eng., vol. 63, pp. 530-539, 2016.

[20] E. Porter, H. Bahrami, A. Santorelli, B. Gosselin, L. A. Rusch, and M. Popović, "A Wearable Microwave Antenna Array for Time-Domain Breast Tumor Screening," IEEE Trans. Med. Imag., vol. 35, pp. 15011509, 2016.

[21] J. D. Shea, P. Kosmas, S. C. Hagness, and B. D. Van Veen, "Contrastenhanced microwave imaging of breast tumors: A computational study using 3D realistic numerical phantoms," Inverse Problems, vol. 26, p. 22, 2010 .

[22] P. M. Meaney, A. H. Golnabi, N. R. Epstein, S. D. Geimer, M. W. Fanning, J. B. Weaver, et al., "Integration of microwave tomography with magnetic resonance for improved breast imaging," Med. Phys., vol. 40, pp. -, 2013.

[23] C. B. Top and N. G. Gencer, "Harmonic Motion Microwave Doppler Imaging: A Simulation Study Using a Simple Breast Model," IEEE Trans. Med. Imag., vol. 33, pp. 290-300, 2014.

[24] R. Scapaticci, G. Bellizzi, I. Catapano, L. Crocco, and O. M. Bucci, "An Effective Procedure for MNP-Enhanced Breast Cancer Microwave Imaging," IEEE Trans. Biomed. Eng., vol. 61, pp. 1071-1079, 2014

[25] G. Bellizzi, O. M. Bucci, and I. Catapano, "Microwave Cancer Imaging Exploiting Magnetic Nanoparticles as Contrast Agent," IEEE Trans. Biomed. Eng, vol. 58, pp. 2528-2536, 2011.

[26] X. Wang, T. Qin, R. S. Witte, and H. Xin, "Computational Feasibility Study of Contrast-Enhanced Thermoacoustic Imaging for Breast Cancer Detection Using Realistic Numerical Breast Phantoms," IEEE Trans. Microw. Theory Tech., vol. 63, pp. 1489-1501, 2015.

[27] J. Song, Z. Zhao, J. Wang, X. Zhu, J. Wu, Z. Nie, et al., "Evaluation of Contrast Enhancement by Carbon Nanotubes for Microwave-Induced Thermoacoustic Tomography," IEEE Trans. Biomed. Eng., vol. 62, pp. 930-938, 2015.

[28] M. T. Bevacqua and R. Scapaticci, "A Compressive Sensing Approach for 3D Breast Cancer Microwave Imaging With Magnetic Nanoparticles as Contrast Agent," IEEE Trans. Med. Imag., vol. 35, pp. 665-673, 2016.

[29] D. Liu, K. Gang, L. Ling, C. Ye, S. Vasudevan, W. Joines, et al., "Electromagnetic time-reversal imaging of a target in a cluttered environment," IEEE Trans. Antennas Propag., vol. 53, pp. 3058-3066, 2005 .

[30] P. Kosmas and C. M. Rappaport, "Time reversal with the FDTD method for microwave breast cancer detection," IEEE Trans. Microw. Theory Tech., vol. 53, pp. 2317-2323, 2005.

[31] P. Kosmas and C. M. Rappaport, "FDTD-based time reversal for microwave breast cancer Detection-localization in three dimensions," IEEE Trans. Microw. Theory Tech., vol. 54, pp. 1921-1927, 2006.

[32] Y. Chen, E. Gunawan, L. Kay Soon, W. Shih-chang, K. Yongmin, and S. Cheong-Boon, "Pulse Design for Time Reversal Method as Applied to Ultrawideband Microwave Breast Cancer Detection: A TwoDimensional Analysis," IEEE Trans. Antennas Propag., vol. 55, pp. 194-204, 2007.

[33] Y. Chen, E. Gunawan, K. S. Low, S. c. Wang, C. B. Soh, and T. C. Putti, "Time-reversal ultrawideband breast Imaging: pulse design criteria considering multiple tumors with unknown tissue properties," IEEE Trans. Antennas Propag., vol. 56, pp. 3073-3077, 2008. 
[34] M. D. Hossain, A. S. Mohan, and M. J. Abedin, "Beamspace TimeReversal Microwave Imaging for Breast Cancer Detection," IEEE Antennas Wireless Propag. Lett., vol. 12, pp. 241-244, 2013.

[35] P. Kosmas and C. M. Rappaport, "A matched-filter FDTD-based time reversal approach for microwave breast cancer detection," IEEE Trans. Antennas Propag., vol. 54, pp. 1257-1264, 2006.

[36] M. E. Yavuz and F. L. Teixeira, "Full time-domain DORT for ultrawideband electromagnetic fields in dispersive, random inhomogeneous media," IEEE Trans. Antennas Propag., vol. 54, pp. 2305-2315, 2006.

[37] M. D. Hossain and A. S. Mohan, "Coherent time-reversal microwave imaging for the detection and localization of breast tissue malignancies," Radio Sci., vol. 50, p. 2014RS005553, 2015.

[38] G. Shi and A. Nehorai, "A Relationship Between Time-Reversal Imaging and Maximum-Likelihood Scattering Estimation," IEEE Trans. Signal Process., vol. 55, pp. 4707-4711, 2007.

[39] M. E. Yavuz and F. L. Teixeira, "Space Frequency Ultrawideband TimeReversal Imaging," IEEE Trans. Geosci. Remote Sens., vol. 46, pp. $1115-1124,2008$.

[40] A. E. Fouda and F. L. Teixeira, "Statistical Stability of Ultrawideband Time-Reversal Imaging in Random Media," IEEE Trans. Geosci. Remote Sensing, vol. 52, pp. 870-879, 2014.

[41] H. Ammari, E. Iakovleva, G. Perruson, and D. Lesselier, "MUSIC-type electromagnetic imaging of a collection of small three-dimensional inclusions," SIAM J. Sci. Comp., vol. 29, pp. 674-709, 2007.

[42] Y. Jin and J. M. F. Moura, "Time-Reversal Detection Using Antenna Arrays," Signal Processing, IEEE Transactions on, vol. 57, pp. 1396$1414,2009$.

[43] H. Hung and M. Kaveh, "Focussing matrices for coherent signalsubspace processing," IEEE Trans. Acoust. Speech Sig. Process., vol. 36, pp. 1272-1281, 1988.

[44] M. D. Zoltowski and T.-S. Lee, "Maximum likelihood based sensor array signal processing in the beamspace domain for low angle radar tracking," IEEE Trans. Signal Process., vol. 39, pp. 656-671, 1991.

[45] Z. Wang, J. Li, and R. Wu, "Time-delay- and time-reversal-based robust capon beamformers for ultrasound imaging," IEEE Trans. Med. Imag., vol. 24, pp. 1308-1322, 2005.

[46] A. Taflove and S. C. Hagness, Computational Electrodynamics: The Finite-difference Time-domain Method: Artech House, 2005.

[47] UWCEM breast phantom repository. Available: http://uwcem.ece.wisc.edu/MRIdatabase/index.html

[48] Z. Wanjun and F. Chin, "Entropy-based time window for artifact removal in UWB imaging of breast cancer detection," IEEE Signal Process. Lett., vol. 13, pp. 585-588, 2006.

[49] A. Lazaro, D. Girbau, and R. Villarino, "Wavelet-based breast tumor localization technique using a UWB radar," Progress in Electromagnetic Research, vol. 98, pp. 75-95, 2009.

[50] H. L. V. Trees, Detection, Estimation, and Modulation Theory, Part I. New York: Wiley, 2001.

[51] Y. Bucris, I. Cohen, and M. A. Doron, "Bayesian Focusing for Coherent Wideband Beamforming," IEEE Trans. Audio Speech Language Process., vol. 20, pp. 1282-1296, 2012.

[52] L. Li, W. Zhang, and F. Li, "A Novel Autofocusing Approach for RealTime Through-Wall Imaging Under Unknown Wall Characteristics," IEEE Trans. Geosci. Remote Sensing, vol. 48, pp. 423-431, 2010.

[53] G. H. Golub and C. F. V. Loan, Matrix Computations, Third ed. Baltimore and London: Johns Hopkins Press, 1996.

[54] C. P. Mathews and M. D. Zoltowski, "Eigenstructure techniques for 2-D angle estimation with uniform circular arrays," IEEE Trans. Signal Process., vol. 42, pp. 2395-2407, 1994.

[55] M. D. Zoltowski and L. Ta-Sung, "Beamspace ML bearing estimation incorporating low-angle geometry," IEEE Trans. Aerospace Electron. Sys., vol. 27, pp. 441-458, 1991.

[56] J. Bourqui and E. C. Fear, "System for Bulk Dielectric Permittivity Estimation of Breast Tissues at Microwave Frequencies," IEEE Trans. Microw. Theory Tech., vol. 64, pp. 3001-3009, 2016.

[57] A. J. Devaney, "Time reversal imaging of obscured targets from multistatic data," IEEE Trans Antennas Propag., vol. 53, pp. 1600-1610, 2005.

[58] G. Shi and A. Nehorai, "Cramer-Rao Bound Analysis on Multiple Scattering in Multistatic Point-Scatterer Estimation," IEEE Trans. Signal Process., vol. 55, pp. 2840-2850, 2007.

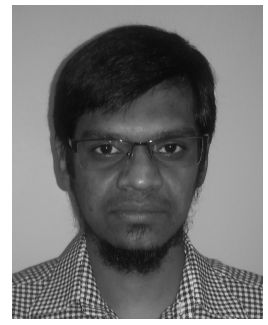

Md. Delwar Hossain (S'11-M'16) received the B.Sc. in electrical and electronic engineering from Rajshahi University of Engineering \& Technology (RUET), Bangladesh, in 2007 and the Ph.D. degree in electrical engineering from University of Technology Sydney (UTS), NSW, Australia, in 2015.

From 2007 to 2010, he was a Lecturer with the electronics and telecommunication engineering department, RUET, Bangladesh. Since 2016, he has been a Learning and Teaching Adjunct with the Faculty of Engineering and IT, UTS, Australia. His research interest includes microwave imaging, inverse scattering, array signal processing, localization, and biomedical applications of microwave.

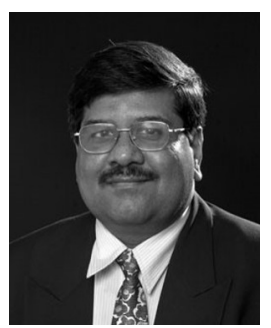

Ananda Mohan Sanagavarapu (aka 'Ananda S Mohan') (SM'05) received the Ph.D. degree from the Indian Institute of Technology, Kharagpur, India. $\mathrm{He}$ is currently an Associate Professor in the recently established School of Biomedical Engineering, Faculty of Engineering and Information Technology, University of Technology Sydney (UTS), NSW, Australia, where he leads research on Applied Electromagnetics, RF and Microwave Thermal Therapies, Implantable and Ingestible Biomedical Devices, Cancer Detection, Microwave Imaging, Array Signal Processing, Wireless Biomedical Communications and Molecular Communications. He is a founding core member of the Interdisciplinary Research Centre on Health Technologies at UTS. He was formerly the Co-Director of the UTS branch of Sydney Microwave Design Resource Centre and also an Associate Director of the UTS node of the multi university Co-operative Research Centre on Satellite Systems. Before joining UTS, he held a postdoctoral fellowship at the Air Navigation Research Group, School of Electrical Engineering at the University of Sydney, Australia. He received a number of competitive research grants from Australian Research Council, National Health and Medical Research Council, and industry. He has also mentored large number of $\mathrm{PhD}$ and masters (research) students. Prof. Mohan was a co-recipient of the Priestly Memorial Award from the Institute of Radio and Electronic Engineers, Australia. He was a member of the IEEE in New South Wales Section Committee and was the past chair of IEEE NSW AP/MTT joint chapter. He was member of technical programme committee of IEEE Globecom-98, a publicity co-chair of the 2011 Asia Pacific Microwave Conference and a Technical Programme Committee Co-Chair for iWAT2014 and ISAP2015. He has been on the international advisory committee for a number of international conferences. 